\title{
Decay mild solutions of fractional differential hemivariational inequalities
}

\author{
xiuwen $\mathrm{li}^{1}$ and Zhenhai $\mathrm{Liu}^{2}$ \\ ${ }^{1}$ Baise University \\ ${ }^{2}$ Central South University
}

August 11, 2020

\begin{abstract}
In this paper, we consider fractional differential hemivariational inequalities (FDHVIs, for short) in the framework of Banach spaces. The aim of this paper is three folds. The first one is to investigate the existence of mild solutions for FDHVIs and by means of a fixed point technique we are able to avoid the hypothesis of compactness on the semigroup. The second aim is to study the existence of decay mild solutions for FDHVI via giving asymptotic behavior of Mittag-Leffler function. Finally, a mathematical model is provided to illustrate our abstract results.
\end{abstract}

\section{Hosted file}

20DMS-FDHVI1.pdf available at https://authorea.com/users/350293/articles/475163-decay-mildsolutions-of-fractional-differential-hemivariational-inequalities 